\title{
Chondrogenesis just ain't what it used to be
}

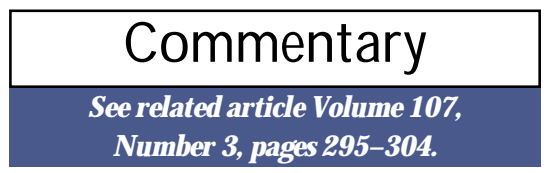

\author{
Gerard Karsenty
}

Department of Molecular and Human Genetics, Baylor College of M edicine, Houston, Texas 77030-4009, USA.

Phone: (713) 798-5489; Fax: (713) 798-1465; E-mail: karsenty@bcm.tmc.edu.

\begin{abstract}
Most of the vertebrate skel eton forms by endochondral ossification, a multistep process that involves two successive cell-differentiation processes. Initially, following mesenchymal cell condensation, a cartilaginous scaffold forms that is later replaced by bone matrix. Under the control of transcription factors of the Sox family, these mesenchymal cells differentiate first into type ll collagen-producing cells that proliferate until the shape of the future bone is established $(1,2)$. Subsequently, cells located at the center of this structure differentiate further to form hypertrophic chondrocytes, which synthesize a cartilaginous matrix that eventually calcifies. At the same time, the most mature hypertrophic chondrocytes undergo apoptosis, al lowing the corresponding area to be invaded by blood vessels, and both multinucleate, matrix-resorbing cells and differentiated osteoblasts appear. The cartilaginous matrix is then degraded and replaced by a bone matrix secreted by osteoblasts.
\end{abstract}

Once this process is completed, the only cartilaginous structures remaining are the growth plate cartilages at the two distal ends of the bone. There, chondrocytes at each stage of differentiation - resting, proliferating, and hypertrophic chondrocytes - are arrayed in a linear succession from the most distal to the most medial regions. This growth plate cartilage therefore offers a powerful model for studying cell differentiation in situ.

Two mechanisms have been generally proposed to explain how chondrocytes and osteoblasts could emerge successively from thesamemesenchymal condensation. First, two populations of progenitors might coexist within the condensations and differentiate al ong separatelineages into chondrocytes and osteoblasts. Alternatively, the samecells could differentiate along a single lineage, first generating chondrocytes, and later differentiating into osteoblasts. A second, related issue concerns the genetic regulation of the different steps of endochondral ossification, chondrogenesis, and bone morphogenesis. M ostly based on mouse genetic experiments, a number of recent studies, including the paper by Chung et al. (3) in a recent issue of the $\mathrm{Jl}$, have established the cascade of events triggering each of these processes and haveidentified key factors required in their regulation (Figure 1).

Hypertrophic chondrocytes and Indian hedgehog in skeletal

formation

The first step toward a molecular understanding of the control of endochondral ossification came from a study designed to reveal the function of the parathyroid hormone-related peptide (PTH rP) in vivo. Karaplis et al. generated PTH rP-deficient mice and observed that they exhibit a generalized abnormality of all skeletal ele ments formed through endochondral ossification. Underlying this defect are a decrease in chondrocyte proliferation and an increase in the rate of chondrocyte hypertrophy in the skeletal condensations (4). Subsequently, two other landmark studies established that the balance between chondrocyte proliferation and chondrocyte hypertrophy is controlled by a negative feedback loop involving two growth factors, PTHrP and Indian hedgehog (Ihh) $(5,6)$. They showed that PT H rP, secreted by the chondrocytes of the perichondrium, signals to the PTH/PTHrP receptor (PPR) expressed on prehypertrophic chondrocytes to suppress their differentiation into hypertrophic chondrocytes. Conversely, prehypertrophic chondrocytes secrete Ihh, which signals to the cells of perichondrium and upregulates their synthesis of PTH rP, thereby indirectly slowing the pace of chon- drocyte hypertrophy. Schipani et al. extended these studies, demonstrating that the chondrocyte-specific, constitutive expression of PPR in mice causes a delay in conversion of the proliferative chondrocyte into the hypertrophic chondrocyte. This phe notype mimics the short-limb dwarfism seen in the genetic disorder Jansen metaphyseal chondrodysplasia, which is caused by activating mutations in the human PPR gene $(7,8)$.

This first set of studies also brought to light a possible link between the presence of hypertrophic chondrocytes and vascular invasion. This direct relationship was demonstrated by the analysis of G elatinase B (M M P-9)-deficient mice, with which Vu et al. demonstrated that cartilage resorption is achieved by chondroclasts, multinucleate cells related to osteoclasts, and that degradation of the cartilaginous matrix regulates vascular invasion via the release of an angiogenic factor (9). Two complementary studies later established that this factor is VEGF, that VEGF is secreted by hypertrophic chondrocytes, and that it acts as a major inducer of vascular invasion $(10,11)$. Because the chondrocyte-specific inactivation of VEGF-A allows for some residual vascularization (11), it appears that another VEG F-likefactor or a cell type distinct from chondrocytes must contribute to the control of vascular invasion during skeletogenesis.

Another major step in our understanding of thegenetic control of endochondral boneformation is seen in the analyses of thelhh-deficient phenotype by St.-Jacques et al. (12) and Chung et al. (3). The former group identified defects affecting both the cartilage formation and osteoblast differentiation and demonstrated that Ihh is not only required for chondrocyte hypertrophy, but also for expression of Cbfal, a transcription factor required for osteoblast differentiation. In mutant animals, 


\section{Figure 1}

M olecular control of endochondral ossification. Transcription factors ( $\mathrm{Cbfal}$ and others) promote the differentiation of the type II collagen-producing cells present in the skeletal mesenchymal condensations into Ihh-secreting hypertrophic chondrocytes. Ihh then acts both on the cells of the perichondrium and on the cells of the bone collar. In the perichondrium, Ihh favors the production of PTH rP, which in turn inhibits chondrocyte hypertrophy. In the bone collar, Ihh induces the expression of Cbfal, which triggers the osteoblastic differentiation of these cells. Hypertrophic chondrocytes also secrete VEGF, which promotes vascular invasion of the skeletal structure. Blood clast/osteoclasts and resorb the ossified cartilagicollar replace this matrix with a bone matrix rich in type I collagen. vessels reach the hypertrophic area, chondronous matrix. O steoblasts derived from the bone

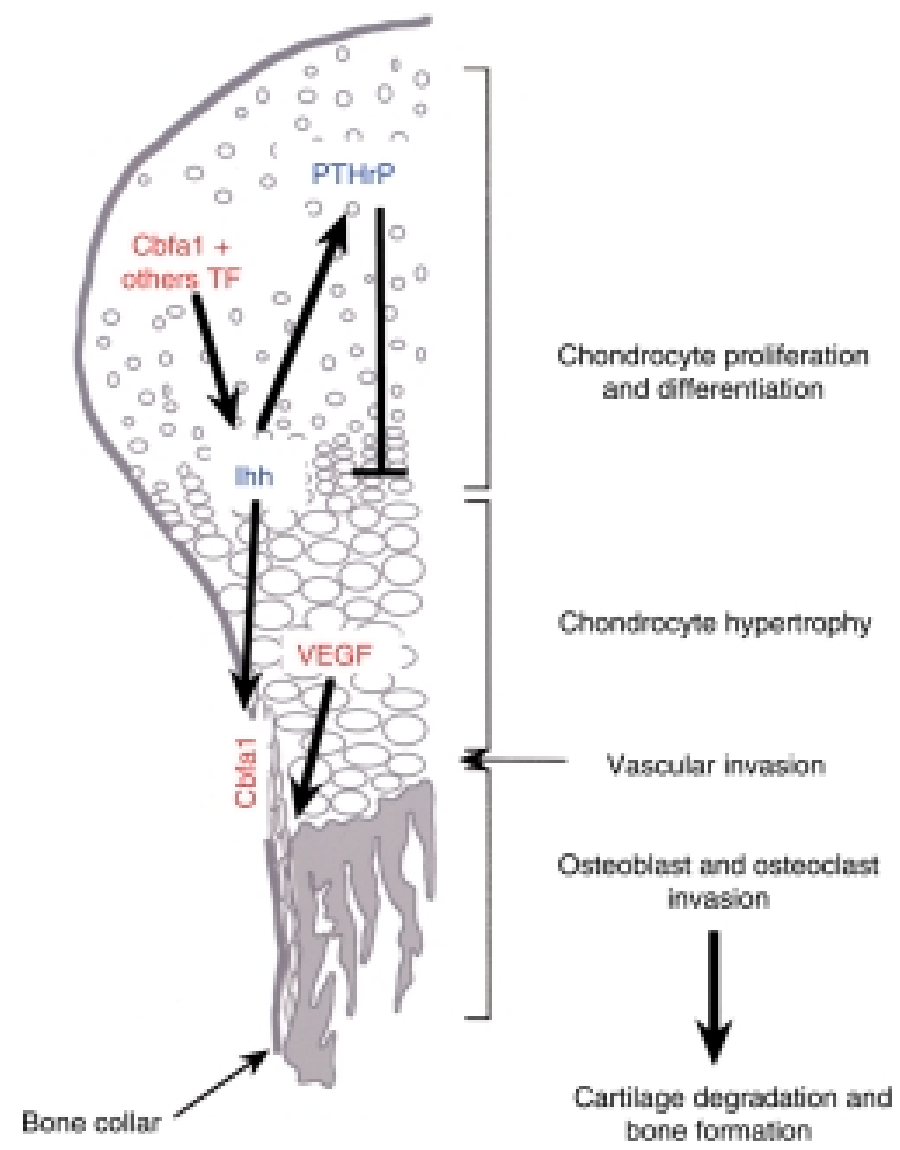

Cbfal is not induced as usual in the cells near the hypertrophic area that line the periphery of condensation. This area corresponds to the histologically well defined structuretermed the "bone collar," the site where calcified bone matrix is first observed (13). As a result of the Ihh defect, mice lack osteoblasts in their long bones, demonstrating that the cells of thebonecollar, not transdifferentiated chondrocytes, represent the predecessors of osteoblasts in these bones. Vascular invasion is also blocked in these mice, confirming the inductiverole of hypertrophic chondrocytes in this process.

An additional link between Ihh and Cbfal was recently established in the context of chondrocytedifferentiation. D etailed studies of the Cbfal-deficient mice had revealed a block of chondrocyte hypertrophy in some skeletal elements $(14,15)$. Targeted overexpression of Cbfal into prehypertrophic chondrocytes of both wild-type mice and Cbfal-deficient mice revealed that Cbfal controls the differentiation of embryonic chondrocytes into hypertrophic chondrocytes, thereby trigger- ing Ihh expression (16). This function of Cbfal is independent of its osteoblast differentiation function, further arguing against a transdifferentiation of chondrocytes into osteoblasts. As this process is defectivein only a few skeletal elements of theCbfal-deficient mice, theremust beother transcription factors that carry out this function elsewherein the skeleton.

Lastly, these studies had together raised a hypothesis that is now confirmed by the elegant study of Chung et al. (3). By comparing chimeric micegenerated with PPR-deficient, or PPR and Ihh doubly deficient cells, this study demonstrates that the distance between Ihh-producing cells and PTHrP-producing cells controls the site of chondrocyte hypertrophy as well as the site of bone collar formation. This finding provides decisive confirmation of the pivotal role played by Ihh and hypertrophic chondrocytes in controlling endochondral ossification.

Future directions

With the development of various null and transgenic animals our under- standing of endochondral bone formation has been transformed. Not only is the cascade of events now established experimentally, but key regulators have been identified that control each step. Still lacking are molecular links between these different factors. For instance, it is unknown whether the PTHrP and Cbfal genes are directly regulated at the transcriptional level by Ihh or Gli, or whether their regulation is indirect. Likewise, it will beimportant to determine whether Cbfal or other transcription factors directly regulate I hh or VEGF gene expression. However, biochemists and cell biologists should now be in a position to resolve these questions and take the next steps toward understanding skel etogenesis.

1. Lefebvre, V., Li, P., and de Crombrugghe, B. 1998. A new long form of Sox5 (L-Sox5), Sox6 and Sox9 are co-expressed in chondrogenesis and cooperatively activate the type II collagen gene. EMBO J. 17:5718-5733.

2.Bi, W., Deng, J.M., Zhang, Z., Behringer, R.R. and deCrombrugghe, $B$. 1999. Sox9 is required for cartilage formation. N at. Genet. 22:85-89.

3. Chung, U., Schipani, E., M CM ahon, A.P., and Kronenberg, H.M. 2001. Indian hedgehog couples 
chondrogenesis to osteogenesis in endochondral bone development. J. Clin. Invest. 107:295-304.

4. Karaplis, A.C., et al. 1994. Lethal skeletal dysplasia from targeted disruption of the parathyroid hormone-related peptide gene. Genes Dev. 8:277-289.

5. Lanske, B., et al. 1996. PTH/PTH rP receptor in early development and Indian hedgehog-regulated bone growth. Science. 273:663-666.

6. Vortkamp, A., et al. 1996. Regulation of rate of cartilage differentiation by Indian hedgehog and PTH -related protein. Science. 273:613-622.

7. Schipani, E., et al. 1997. Targeted expression of constitutively active receptors for parathyroid hormone and parathyroid hormonerelated peptide delays endochondral bone formation and rescues mice that lack parathyroid hormonerelated peptide. Proc. N atl. Acad. Sci. USA.
94:13689-13694

8. Schipani, E., Kruse, K., and Juppner, H. 1995. A constitutively active mutant PTH -PTH rP receptor in Jansen-type metaphyseal chondrodysplasia. Science. 268:98-100.

9. Vu, T.H., et al. 1998. M M P-9/gelatinase B is a key regulator of growth plate angiogenesis and apoptosis of hypertrophic chondrocytes. Cell. 93:411-422.

10. Gerber, H.P., et al. 1999. VEGF couples hypertrophic cartilage remodeling, ossification and angiogenesis during endochondral bone formation. Nat. M ed. 5:623-628.

11. Haigh, J.J., Gerber, H.-P., Ferrara, N ., and Wagner, E.F. 2000. Conditional inactivation of VEGF-A in areas of collagen2A1 expression results in embryonic lethality in the heterozygous state. D evelopment. 127:1445-1453.

12. St.-Jacques, B., Hammerschmidt, M., and
M CM ahon, A.P. 1999. Indian hedgehog signaling regulates proliferation and differentiation of chondrocytes and is essential for bone formation. Genes Dev. 13:2072-2086.

13. Caplan, A.I., and Pechak, D.G. 1987. The cellular and molecular embryology of bone formation. Elsevier. N ew York, N ew York, USA. 117-183.

14. Kim, I.S., Otto, F., Abel, B., and Mundlos, S 1999. Regulation of chondrocyte differentiation by Cbfa1. M ech. Dev. 80:159-170.

15. Inada, M., et al. 1999. M aturational disturbance of chondrocytes in Cbfal-deficient mice. Dev. Dyn. 214:279-290.

16. Takeda, S., Bonnamy, J.P., O wen, M .J., D ucy, P., and Karsenty, G. 2001. Continuous expression of Cbfal in non-hypertrophic chondrocytes uncovers its ability to induce hypertrophic chondrocytes differentiation and partially rescues Cbfal-deficient mice. Genes Dev. In press. 